\title{
NUEVOS REQUISITOS PARA EL PERFIL DEL EJECUTIVO ACTUAL
}

Alfonso Mujica*

* Director Ejecutivo de Spencer Stuart en Chile. 


\section{RESUMEN}

Los requisitos para ocupar cargos ejecutivos en las empresas e instituciones han sufrido un cambio radical en las últimas décadas y especialmente en los últimos años. En su evolución, incorporan cada vez más y en forma prioritaria los factores vinculados con la actitud, tales como la honorabilidad, el liderazgo, la capacidad de interacción social e influencia, la productividad y energía vital, el autocontrol y la perseverancia, así como el equilibrio y la consecuencia. No por ello disminuye la importancia de los conocimientos, ya que constituyen la base del aprendizaje. En mi opinión, esto plantea un desafío para las universidades, que implica mucho más que un cambio en los programas de cursos, un cambio en la forma de enseñar. Los talleres y ejercicios repetitivos son irreemplazables cuando se quiere producir cambios de hábitos.

\section{ABSTRACT}

Requirements to hold executive positions in companies and organizations have undergone a radical change in the last decades -particularly in the past few years. They increasingly and significantly tend to include factors involving the individuals' personal qualities, such as integrity, leadership capabilities, social skills and ability to exert influence, in addition to productivity and energy, self-control and perseverance, as well as a balanced and consistent personality. This does not mean that the importance of knowledge has diminished, since it is the basis of learning. This raises a challenge for universities, that calls for more than a simple change in curricula and requires, instead, a change in teaching methods. Workshops and repetitive exercises are irreplaceable when the desired outcome is a change in habits. 


\section{NUEVOS REQUISITOS PARA EL PERFIL DEL EJECUTIVO ACTUAL}

Los requisitos para ocupar cargos ejecutivos en las empresas e instituciones han ido evolucionando radicalmente en las últimas décadas y especialmente en los últimos años. Ello tiene suma importancia a la hora de definir el contenido de los programas de educación superior para las carreras profesionales.

En los tiempos actuales podríamos decir que la clave está en la flexibilidad, en comparación con el énfasis en la enseñanza de técnicas más "permanentes" que se proclamaba históricamente.

Si analizamos las técnicas aprendidas en la Universidad por quienes hoy ocupan altas posiciones ejecutivas, veremos que están casi totalmente obsoletas. Si preguntamos al típico "gerente general" de nuestros días respecto de las técnicas sobre las cuales se hizo mayor hincapié durante su época de universidad, nos dirá que fueron los "procesos de optimización" si es ingeniero y la "corrección monetaria y procesos inflacionarios" si es ingeniero comercial. Actualmente, ambos conocimientos resultan perfectamente inútiles, pues quedaron superados por la tecnología y por la historia. ${ }^{1}$

De igual modo, lo que estamos enseñando a nuestros alumnos de hoy será con seguridad obsoleto cuando ellos lleguen a ocupar cargos de importancia en las empresas e instituciones del mañana.

Por ello, hoy más que nunca cobra importancia la formación general que permita al alumno desarrollar un modelo de la realidad sobre el cual desarrollar aplicaciones a su futura "realidad profesional".

1. En mi situación de consultor y seleccionador de los ejecutivos que contratan las empresas, me encuentro permanentemente ante la necesidad de discutir con mis clientes el perfil de los candidatos a ocupar posiciones ejecutivas. Al estudiar los requisitos que se solicitan, me llama la atención que la mayoría se vincula a la actitud del candidato, mientras que los conocimientos constituyen un valor en la medida en que se reflejan en su trayectoria y logros profesionales. 
Esta realidad plantea un nuevo desafío para las universidades, pues ya no triunfa el ejecutivo basado sólo en sus conocimientos y que impone su autoridad a su gusto en una estructura vertical. Las empresas son, en este momento, organizaciones a menudo de estructuras matriciales y de funcionamiento democrático, donde no opera la autoridad sino la influencia.

Ello no significa en ningún caso que considere necesario disminuir la adquisición de conocimientos, aunque éstos puedan quedar obsoletos, puesto que constituyen una base de formación intelectual imprescindible. Pero debemos sumar a ello la necesidad de enseñar actitudes y hábitos, lo cual requiere el uso de otras técnicas de aprendizaje. He sido testigo de varios intentos frustrados en este sentido. Esto se debe, fundamentalmente, al hecho de que se sigue usando el sistema de clases expositivas, en tediosas sesiones que apuntan a la intelectualización en vez de a la generación de hábitos. Estos sólo pueden ser adquiridos en talleres que, a través de una constante repetición, logren incorporar dichos hábitos y actitudes en la "biología" del alumno.

Los requerimientos en materia de características personales y profesionales que formulan las empresas para los ejecutivos que contratan, podrían sintetizarse de la siguiente manera:

Honorabilidad a toda prueba, demostrable a través de una intachable carrera profesional. Debido a lo subjetivo del tema resulta difícil de abordar en el plano de la enseñanza, pero la formación valórica desempeña un papel importante, especialmente a fin de poder anticipar y enfrentar situaciones que, en este plano, puedan presentarse en su futura vida profesional.

Liderazgo, que implica ser capaz de hacer productivo el tiempo de las personas que supervisa, no sólo en el corto plazo, sino que en forma permanente. Por inteligente y capaz que sea un profesional trabajando solo, no es de interés de las empresas si no tiene la capacidad de acicatear la productividad de los demás, dirigiéndolos y motivándolos efectivamente. En esta materia, el éxito está muy influenciado por las características de personalidad del profesional, entre ellos y muy fundamentalmente la empatía. Sin embargo, es 
posible generar hábitos orientados a mejorar la efectividad del liderazgo.

Capacidad de interacción social e influencia en el medio externo a la empresa. Hoy ya no sirve el ejecutivo aislado del mundo y encerrado en su oficina. La capacidad de interactuar positivamente con autoridades, clientes, proveedores, competidores y la sociedad en general, es hoy de vital importancia. Recuerdo que en la primera empresa en la cual trabajé propuse contratar una firma externa para que nos proporcionara el servicio de vigilancia. Tuve que escuchar una extensa argumentación por parte del directorio, respecto de por qué resultaba más económico y eficiente hacerlo todo desde el interior de la propia empresa. En la actualidad nadie discute la conveniencia de externalizar los servicios y las empresas se han transformado, de hecho, en una red de cooperación con clientes y proveedores internos y externos, que interactúan en pos de un objetivo compartido. Por ello, el éxito de las empresas está marcado cada vez más por la capacidad de influir e interactuar de sus ejecutivos.

Productividad y energía vital, que proporcione energía y motivación a todo el grupo humano que allí trabaja. Se ha comprobado que la productividad depende directamente de la capacidad que tenga el líder para inspirar a sus subordinados. Recientemente, analizábamos durante un proceso de selección a dos candidatos; uno de ellos, con experiencia y capacidad técnica perfectamente congruente con el perfil diseñado para el cargo. Sin embargo, el seleccionado fue un ejecutivo cuya experiencia no cubría los requerimientos del cargo, pero sus niveles de energía y su interés por el logro convencieron al directorio, el cual consideró que estos factores más que compensaban su déficit. Si bien es difícil enseñar esta actitud, es posible motivarla y valorarla en la universidad.

Autocontrol y perseverancia: la forma de operar de las empresas en la actualidad, con estructuras menos jerárquicas y muchas veces matriciales, entrega cada vez más autonomía a los ejecutivos. Por esta razón, deben ser capaces de automotivarse y autocontrolarse, perseverando en la búsqueda del logro de sus objetivos. Mientras más alta sea la posición de un ejecutivo, menos clara es su línea de reporte y por consiguiente más importante resultan su capacidad de 
automotivarse y autocontrolarse. Lamentablemente el sistema de enseñanza de clases expositivas y pruebas no contribuye en nada para desarrollar estas actitudes.

Equilibrio y consecuencia: una persona equilibrada y consecuente es capaz de construir confianza con su equipo, sus clientes y proveedores y con todos los intereses de la empresa. Ello tiene implicancia en la carrera profesional y en la vida personal. Mi experiencia me dice que, a la hora de seleccionar ejecutivos, siempre importa cuán consecuente es la persona en los distintos aspectos de su vida, desde que posea una carrera profesional estable pero ascendente, hasta que su vida personal sea ordenada.

En relación con los requisitos técnicos, es más difícil generalizar. Sin embargo es fundamental el desarrollo de la capacidad de aprender y de modelar. En general las demás técnicas, si no se han adquirido durante los estudios, se aprenden en los primeros años de vida laboral. Hay expertos en sistemas contables o bien contralores que son ingenieros civiles; gerentes de informática que son ingenieros comerciales o gerentes de recursos humanos que son abogados. Aunque sus respectivas formaciones en contabilidad, sistemas o recursos humanos no sean las más profundas, lo importante es el aprendizaje de una disciplina de pensamiento que les permita aprender de ahí en adelante.

La mayoría de los altos ejecutivos salieron de la universidad antes que existieran las calculadoras de bolsillo o los computadores personales, no conocían las fotocopiadoras ni los fax, ni menos Internet, y han debido aplicar métodos y sistemas que no pudieron aprender en la universidad. Son avances que han cambiado radicalmente la forma de hacer negocios y de manejar las empresas. Cada día es más importante la flexibilidad para aprender cosas nuevas, pues la velocidad del cambio es creciente.

Finalmente quisiera comentar respecto al aprendizaje del idioma inglés. La búsqueda de ejecutivos plantea en un $97 \%$ de los casos el requerimiento de conocer bien dicho idioma. Lamentablemente, la mayoría de los ejecutivos tiene serias limitaciones en este aspecto. Hoy día, ello ha cobrado una importancia similar al dominio de los lenguajes técnicos de cada profesión. 